\title{
On existence of minimizers for the Hardy-Sobolev-Maz'ya inequality
}

\author{
A.Tertikas \\ Department of Mathematics \\ University of Crete \\ 71409 Heraklion, Greece, and \\ Institute of Applied and Computational Mathematics \\ FORTH, 71110 Heraklion, Greece \\ tertikas@math.uoc.gr
}

\author{
K.Tintarev \\ Department of Mathematics \\ Uppsala University \\ SE-751 06 Uppsala, Sweden \\ tintarev@math.uu.se
}

November 9, 2018

\begin{abstract}
We show existence of minimizers for the Hardy-Sobolev-Maz'ya inequality in $\mathbb{R}^{m+n} \backslash \mathbb{R}^{n}$ when either $m>2, n \geq 1$ or $m=1, n \geq 3$.
\end{abstract}

The authors expresses their gratitude to the faculties of mathematics departments at Technion - Haifa Institute of Technology, at the University of Crete and of the University of Cyprus for their hospitality. A.T. acknowledges partial support by the RTN European network Fronts-Singularities, HPRN-CT-200200274. K.T acknowledges support as a Lady Davis Visiting Professor at Technion and partial support from University of Crete, University of Cyprus and Swedish Research Council.

Mathematics Subject Classifications: 35J65, 35J20, 35J70.

Key words: singular elliptic operators, semilinear elliptic equations, Hardy inequality, critical exponent, concentration compactness, Brezis-Nirenberg problem 


\section{Introduction}

Let $N=n+m \geq 3, n=0, \ldots, N-1$. The space $\mathbb{R}^{N}=\mathbb{R}^{m+n}$ will be regarded here as a product $\mathbb{R}^{n} \times \mathbb{R}^{m}$ and the variables in $\mathbb{R}^{N}$ will be denoted as $(x, y), x \in \mathbb{R}^{n}, y \in \mathbb{R}^{m}$. In this notations, the Hardy inequality involving the distance from $\mathbb{R}^{n} \times\{0\}$ (which will be for brevity denoted as $\mathbb{R}^{n}$ ) reads

$$
\int_{\mathbb{R}^{n+m}}|\nabla u(x, y)|^{2} d x d y \geq\left(\frac{m-2}{2}\right)^{2} \int_{\mathbb{R}^{n+m}} \frac{u^{2}(x, y)}{|y|^{2}} d x d y, \quad u \in C_{0}^{\infty}\left(\mathbb{R}^{n+m} \backslash \mathbb{R}^{n}\right) .
$$

The constant $\left(\frac{m-2}{2}\right)^{2}$ that appears in (11) is the best constant and is not attained. Maz'ya [13], Corollary 3, p. 97] was the first that discovered that an additional term with the critical Sobolev exponent $2^{*}=\frac{2 N}{N-2}$ can be added in the right hand side. That is, when $n \neq 0$, the following Hardy-Sobolev-Maz'ya inequality holds true:

$$
\int_{\mathbb{R}^{n+m}}|\nabla u(x, y)|^{2} d x d y-\left(\frac{m-2}{2}\right)^{2} \int_{\mathbb{R}^{n+m}} \frac{u^{2}(x, y)}{|y|^{2}} d x d y \geq \kappa_{m, n}\|u\|_{2^{*}}^{2}, \quad u \in C_{0}^{\infty}\left(\mathbb{R}^{n+m} \backslash \mathbb{R}^{n}\right) .
$$

If $m=2$, the inequality (2) becomes the usual limit exponent Sobolev inequality, since in this case $C_{0}^{\infty}\left(\mathbb{R}^{n+m} \backslash \mathbb{R}^{n}\right)$ is dense in $\mathcal{D}^{1,2}\left(\mathbb{R}^{n+m}\right)$. It is worth noting that when $n=0$ and the distance is taken from the origin, the inequality (10) is no longer true (cf. Brezis and Vázquez [5]). Let $\Omega$ is a bounded domain with $0 \in \Omega, X(r)=(1-\log r)^{-1}$ for $0<r \leq 1$, and let $D:=\sup _{x \in \Omega}|x|<+\infty$. Then one has ([16], Theorem A) the following analog of (2):

$$
\int_{\Omega}|\nabla u|^{2} d x-\left(\frac{N-2}{2}\right)^{2} \int_{\Omega} \frac{u^{2}}{|x|^{2}} d x \geq C\left(\int_{\Omega}|u|^{\frac{2 N}{N-2}} X^{\frac{2(N-1)}{N-2}}\left(\frac{|x|}{D}\right) d x\right)^{\frac{N-2}{N}}, \quad u \in C_{0}^{\infty}(\Omega) .
$$

Inequality (3) involves the critical exponent, but contrary to (21) it has a logarithmic correction. Moreover, it is sharp in the sense that one cannot take a smaller power of the logarithmic correction $X$. In this paper our interest is in the existence of minimizers to the Hardy-Sobolev-Maz'ya inequality (2).

In case of $m=1$ the set $\mathbb{R}^{n+m} \backslash \mathbb{R}^{n}$ is disconnected, so the problem can be naturally restated as a problem on the half-space. However, in order to keep the notations uniform, this reduction is not made here. We exclude from consideration the case $m=2$ when the inequality (2) becomes the usual Sobolev inequality with the limit exponent and the case $m=N$ (that is, $n=0$ ) when the inequality does not hold.

Note that the expression

$$
\|u\|_{\mathcal{H}_{0}}^{2}:=\int_{\mathbb{R}^{n+m}}\left(|\nabla u(x, y)|^{2}-\left(\frac{m-2}{2}\right)^{2} \frac{u^{2}(x, y)}{|y|^{2}}\right) d x d y
$$

is a quadratic form, positive definite due to (2) on $C_{0}^{\infty}\left(\mathbb{R}^{n+m} \backslash \mathbb{R}^{n}\right)$, and therefore a scalar product. Also by (21), the Hilbert space $\mathcal{H}_{0}$, defined by completion of $C_{0}^{\infty}\left(\mathbb{R}^{n+m} \backslash \mathbb{R}^{n}\right)$ with respect the norm above is continuously imbedded into $L^{2^{*}}\left(\mathbb{R}^{n+m}\right)$ whenever $n>0$, and the elements of $\mathcal{H}_{0}$ can be identified as measurable functions (modulo a.e.). 
Let $T: C_{0}^{\infty}\left(\mathbb{R}^{n+m} \backslash \mathbb{R}^{n}\right) \rightarrow C_{0}^{\infty}\left(\mathbb{R}^{n+m} \backslash \mathbb{R}^{n}\right)$ be given by

$$
(T v)(x, y)=|y|^{-\frac{m-2}{2}} v(x, y)
$$

and define a Hilbert space $\mathcal{H}$ as a completion of $C_{0}^{\infty}\left(\mathbb{R}^{n+m} \backslash \mathbb{R}^{n}\right)$ under the norm $\|v\|_{\mathcal{H}}:=\|T v\|_{\mathcal{H}_{0}}^{2}$. It is clear that $T$ extends to an isometry between $\mathcal{H}$ and $\mathcal{H}_{0}$ as well as to an isometry between $L^{2^{*}}\left(\mathbb{R}^{n+m},|y|^{2^{*}(1-m / 2)}\right)$ and $L^{2^{*}}\left(\mathbb{R}^{n+m}\right)$. In particular, for $n \neq 0$ the space $\mathcal{H}$ consists of measurable functions. Furthermore, elementary computations show that

$$
\|v\|_{\mathcal{H}}:=\|T v\|_{\mathcal{H}_{0}}^{2}=\int_{\mathbb{R}^{n+m}}|y|^{-(m-2)}|\nabla v|^{2} d x d y,
$$

i.e. $\mathcal{H}=\mathcal{D}^{1,2}\left(\mathbb{R}^{n+m},|y|^{-(m-2)}\right)$, and the inequality (2) takes the equivalent form

$$
\int_{\mathbb{R}^{n+m}}|y|^{-(m-2)}|\nabla v|^{2} d x d y \geq \kappa_{m, n}\left(\int_{\mathbb{R}^{n+m}}|y|^{2^{*}(1-m / 2)}|v|^{2^{*}} d x d y\right)^{2 / 2^{*}} .
$$

We prove the following statement:

Theorem 1.1. The minimization problem

$$
\kappa_{m, n}=\inf _{\int_{\mathbb{R}^{n+m}}|y|^{2^{*}(1-m / 2)}|v|^{2^{*}} d x d y=1} \int_{\mathbb{R}^{n+m}}|y|^{-(m-2)}|\nabla v|^{2} d x d y
$$

has a point of minimum in $\mathcal{H}=\mathcal{D}^{1,2}\left(\mathbb{R}^{n+m},|y|^{-(m-2)}\right)$ whenever $m>2, n>0$ or $m=1, n \geq 3$.

Theorem 1.2. The minimization problem

$$
\kappa_{m, n}=\inf _{u \in \mathcal{H}_{0}: \int_{\mathbb{R}^{n+m}}|u|^{2^{*}} d x d y=1}\|u\|_{\mathcal{H}_{0}}^{2}
$$

has a point of minimum in $\mathcal{H}_{0}$, the completion of $C_{0}^{\infty}\left(\mathbb{R}^{n+m} \backslash \mathbb{R}^{n}\right)$ with respect to the norm (4), whenever $m>2, n>0$ or $m=1, n \geq 3$.

Due to the transformation (5) Theorems 1.1 and 1.2 are equivalent. The minimizer of (9) resp. (8) and the exact value of $\kappa_{m, n}$ remains unknown. Theorems 1.1 and 1.2 do not include the case $m=1$ and $n=2$.

The problem (88) is not compact, and we use a concentration-compactness technique similar to one of [2, based on weak convergence argument and the Brezis-Lieb lemma ([3]). Its application is, however, not straightforward. The group of invariant transformations (which include dilations) that suffices to treat a similar problem in [1] or [19], does not suffice here. In their case, the critical dilation invariance that is caused by a singular weight, rather than by critical growth of nonlinearity, reduces the nonlinear term to a subcritical one, once the domain of the problem is partitioned into similar cells (of varying diameter that goes to zero as the cell approaches the singularity). However, reduction of the term $\int|u|^{2^{*}}$ to a subcritical term requires a partition of both the domain and the range of $u$ into similar compact sets, which makes it inevitable to append the group of available invariant transformations by the non-invariant translations in the $y$-variable. This is possible, but only because the latter incur a variational penalty. 
It might be useful for the reader more accustomed to the P.-L.Lions' version of concentration compactness $([8,[9], 10], 11])$ to give here some heuristic interpretation of the problem in those terms. In the problem (8) four different types of concentration arise: translations in the $x$-variable, translations in the $y$-variable, concentration in the interior and concentration at the boundary (including concentration at infinity). Translations in $\left|y_{k}\right| \rightarrow \infty$ incurs a variational penalty and so does the interior concentration, provided that the infimum value $\kappa_{m, n}$ is less than the Sobolev constant

$$
S_{N}:=\inf _{w \in \mathcal{D}^{1,2}\left(\mathbb{R}^{N}\right):\|w\|_{2^{*}=1}} \int_{\mathbb{R}^{N}}|\nabla w|^{2}, N \geq 3 .
$$

We have established that $\kappa_{m, n}<S_{m+n}$ whenever $m+n>3$. The remaining concentrations, the concentration on the boundary and the translations in $x$, are due to invariant transformations and are handled by the subadditivity argument.

In order to consider the analog of the problem (2) on an open set $\Omega \subset \mathbb{R}^{n+m} \backslash \mathbb{R}^{n}$ we would like to

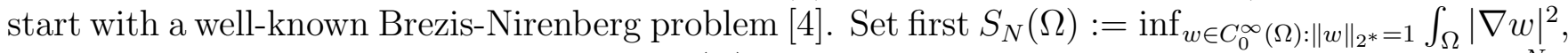
$N \geq 3$. It is well known that for every $\Omega, S_{N}(\Omega)=S_{N}$ and that there is no minimizer when $\Omega \neq \mathbb{R}^{N}$.

In [4] one considers a bounded set $\Omega \subset \mathbb{R}^{N}$ and the minimization problem

$$
S_{\lambda, N}(\Omega):=\inf _{u \in H_{0}^{1}(\Omega):\|w\|_{2^{*}=1}} \int_{\Omega}\left(|\nabla u|^{2}-\lambda u^{2}\right),
$$

where $\lambda>0$ does not exceed the first eigenvalue $\lambda_{1}$ of the Dirichlet Laplacian in $\Omega$. It is shown in [4] that the inequality $S_{\lambda, N}(\Omega)<S_{N}$ (separation of the infimum from the concentration level) holds for $N>3$ ( as well as for $\lambda$ sufficiently close to $\lambda_{1}$ when $N=3$ ), from which existence of the minimizer for $S_{\lambda, N}(\Omega)$ easily follows.

In our case we consider, for an open set $\Omega \varsubsetneqq \mathbb{R}^{n+m} \backslash \mathbb{R}^{n}, n \neq 0, m \neq 2, m+n \geq 3$, the minimization problem

$$
\kappa_{m, n}(\Omega)=\inf _{u \in C_{0}^{\infty}(\Omega): \int_{\Omega}|u|^{2^{*}} d x d y=1} \int_{\Omega}\left(|\nabla u|^{2}-\left(\frac{m-2}{2}\right)^{2} \frac{u^{2}}{|y|^{2}}\right) d x d y .
$$

An equivalent problem under transformation (15) is

$$
\kappa_{m, n}(\Omega)=\inf _{u \in \mathcal{D}^{1,2}\left(\Omega,|y|^{-(m-2)}\right): \int_{\Omega}|y|^{2^{*}(1-m / 2)}|v|^{2^{*}} d x d y=1} \int_{\Omega}|y|^{-(m-2)}|\nabla v|^{2} d x d y
$$

where $\mathcal{D}^{1,2}\left(\Omega ;|y|^{-(m-2)}\right)$ is the completion of $C_{0}^{\infty}(\Omega)$ with respect to the norm $\left(\int_{\Omega}|y|^{-(m-2)}|\nabla v|^{2} d x d y\right)^{\frac{1}{2}}$.

There are still four types of concentration as in the case of $\mathbb{R}^{n+m} \backslash \mathbb{R}^{n}$. Concentration in the interior yields $S_{N}>\kappa_{m, n}(\Omega)$, provided that $N>3$. Concentration at a boundary point with $y=0$ occurs at the energy level $\kappa_{m, n}$. By monotonicity, for any $\Omega, \kappa_{m, n}(\Omega) \geq \kappa_{m, n}$.

It is easy to see that whenever $\kappa_{m, n}(\Omega)=\kappa_{m, n}, \Omega \neq \mathbb{R}^{n+m} \backslash \mathbb{R}^{n}$, the constant $\kappa_{m, n}(\Omega)$ is not attained: if $v$ were a minimizer for $\kappa_{m, n}(\Omega)$, it would be then a minimizer for $\kappa_{m, n}$, contrary to the maximum principle. In particular, 
Theorem 1.3. (Non-existence of minimizers for $\Omega \varsubsetneqq \mathbb{R}^{n+m} \backslash \mathbb{R}^{n}$.) Assume that $m \neq 2, n \neq 0$ and $m+n \geq 3$. Then $\kappa_{m, n}(\Omega)=\kappa_{m, n}$ provided that one of the following conditions holds true:

a) there exist $x_{0} \in \mathbb{R}^{n}$ and $r>0$ such that $\Omega$ contains $B_{r}\left(x_{0}, 0\right) \backslash\{y=0\}$,

b) there exists $R>0$ such that $\Omega$ contains the set $\{|y|>R\}$.

There are also domains where we have existence of a minimizer (consequently $\left.\kappa_{m, n}(\Omega)>\kappa_{m, n}\right)$. We consider existence only for domains $\Omega$ contained in

$$
A_{r, R}=\left\{(x, y) \in \mathbb{R}^{n+m} \backslash \mathbb{R}^{n}, r<|y|<R\right\}, 0<r<R<\infty .
$$

This condition is not a heavy restriction in view of Theorem 1.3. Under this assumption there is no concentration related to translations in $y$. Concentration due to translations in the $x$-variable is handled by subadditivity, under a flask-type assumption on $\Omega$, that is: for every sequence $x_{k} \in \mathbb{R}^{n}$, there exists $x_{0} \in \mathbb{R}^{n}$ such that

$$
\liminf \left(\Omega+x_{k}\right) \subset \Omega+x_{0} .
$$

The existence proof for non-invariant domains cannot use the Brezis-Lieb lemma directly. Instead, following the method of [17], it uses Lemma [5.2]- an "iterated" version of Brezis-Lieb lemma.

Theorem 1.4. Suppose that for some $0<r<R<\infty$,

$$
\Omega \subset A_{r, R}
$$

$\partial \Omega \in C^{1}$ and, in addition, $\Omega$ satisfies (15). Then the minimization problem

$$
\kappa_{m, n}(\Omega)=\inf _{\int_{\Omega}|y|^{2^{*}(1-m / 2)}|v|^{2^{*}} d x d y=1} \int_{\Omega}|y|^{-(m-2)}|\nabla v|^{2} d x d y
$$

attains a minimum in $H_{0}^{1}(\Omega)$ provided that $m>2, n>0$, or $m=1, n \geq 3$.

Remark 1.5. Under hypothesis (16) we have $\mathcal{D}^{1,2}\left(\Omega,|y|^{-(m-2)}\right)=H_{0}^{1}(\Omega)$. This in particular implies existence of the minimizer of (12) in the class $H_{0}^{1}(\Omega)$. This is not the case when $\Omega=$ $\mathbb{R}^{n+m} \backslash \mathbb{R}^{n}$.

In Section 2 we make preliminary computations used later in the proofs. In Section 3 we prove that minimization sequences under unbounded translations in the $y$-variable converge weakly to zero. Section 4 concludes the proof of the main result (Theorems 1.1] and 1.2). In Section 5 we prove Theorems 1.3 and 1.4. In Section 6 we give an alternative proof of the main result and outline some open problems.

In what follows, integration without domains or variables specified will always refer to $\mathbb{R}^{n+m} \backslash \mathbb{R}^{n}$ and $d x d y$, respectively. 


\section{Preliminary computations}

Lemma 2.1. Let $w \in H_{\text {loc }}^{1}\left(\mathbb{R}^{n+m} \backslash \mathbb{R}^{n}\right)$. For every $\epsilon \in\left(1, \frac{1}{4}\right)$ there exists a $w_{\epsilon} \in C_{0}^{\infty}\left(\mathbb{R}^{n+m} \backslash \mathbb{R}^{n}\right)$ such that

$$
\int \nabla w \cdot \nabla w_{\epsilon} \geq(1-\epsilon) \int\left|\nabla w_{\epsilon}\right|^{2}
$$

Moreover, if $w \in \mathcal{D}^{1,2}\left(\mathbb{R}^{n+m} \backslash \mathbb{R}^{n}\right)$ then $w_{\epsilon}$ satisfies, in addition to (18), $\left\|w-w_{\epsilon}\right\|_{\mathcal{D}^{1,2}} \leq \epsilon\|w\|_{\mathcal{D}^{1,2}}$.

Proof. Assume first that $w \in \mathcal{D}^{1,2}\left(\mathbb{R}^{n+m} \backslash \mathbb{R}^{n}\right)$ and let $\epsilon>0$. In this proof we use the notation of the norm and of the scalar product in reference to the space $\mathcal{D}^{1,2}\left(\mathbb{R}^{n+m} \backslash \mathbb{R}^{n}\right)$. By density of $C_{0}^{\infty}\left(\mathbb{R}^{n+m} \backslash \mathbb{R}^{n}\right)$ in $\mathcal{D}^{1,2}\left(\mathbb{R}^{n+m} \backslash \mathbb{R}^{n}\right)$, and since $w \neq 0$, one can choose a $w_{\epsilon} \in C_{0}^{\infty}\left(\mathbb{R}^{n+m} \backslash \mathbb{R}^{n}\right)$ such that $\left\|w-w_{\epsilon}\right\| \leq \epsilon\|w\|$. Using the Cauchy inequality, we have

$$
\left(w, w_{\epsilon}\right)=\|w\|^{2}-\left(w, w-w_{\epsilon}\right) \geq\|w\|^{2}-\|w\|\left\|w-w_{\epsilon}\right\| \geq(1-\epsilon)\|w\|^{2} .
$$

This proves the second assertion of the lemma.

It remains now to consider the case $w \in H_{\text {loc }}^{1}\left(\mathbb{R}^{n+m} \backslash \mathbb{R}^{n}\right) \backslash \mathcal{D}^{1,2}\left(\mathbb{R}^{n+m} \backslash \mathbb{R}^{n}\right) \backslash\{0\}$. Then

$$
\sup _{\psi \in C_{0}^{\infty}(\Omega):\|\psi\|=1}(w, \psi)=+\infty
$$

since the finite value of the supremum yields $w \in \mathcal{D}^{1,2}\left(\mathbb{R}^{n+m} \backslash \mathbb{R}^{n}\right)$. In particular, there exists a $w_{1} \in C_{0}^{\infty}\left(\mathbb{R}^{n+m} \backslash \mathbb{R}^{n}\right),\left\|w_{1}\right\|=1$, such that $\left(w, w_{1}\right)>1$. Set $w_{\epsilon}=w_{1}$.

Lemma 2.2. Let $\Omega \subset \mathbb{R}^{n+m} \backslash \mathbb{R}^{n}$ be an open set. If

(i) $m>2$ and $n \geq 1$, or

(ii) $m=1$ and $n \geq 3$.

then $0<\kappa_{m, n}<S_{m+n}$.

Trivially, $\kappa_{2, n}=S_{2+n}$. We do not know whether, in the remaining case, $\kappa_{2,1}<S_{3}$ or the equality prevails.

Proof. Let $z=(x, y) \in \mathbb{R}^{N}$. The unique minimizer for (10), modulo translations and the scale transformation $w \mapsto \epsilon^{-\frac{N-2}{2}} w(z / \epsilon)$ is the well known Bliss-Talenti solution, a scalar multiple of $w=\left(1+|z|^{2}\right)^{-\frac{N-2}{2}}$.

Case (i). When $m>1, C_{0}^{\infty}\left(\mathbb{R}^{n+m} \backslash \mathbb{R}^{n}\right)$ is dense in $\mathcal{D}^{1,2}\left(\mathbb{R}^{n+m}\right)$. Then, since $\|u\|_{\mathcal{H}_{0}} \leq\|u\|_{\mathcal{D}}^{1,2}$, the space $\mathcal{D}^{1,2}\left(\mathbb{R}^{n+m}\right)$ is continuously imbedded into $\mathcal{H}_{0}$ and for every $u \in \mathcal{D}^{1,2}\left(\mathbb{R}^{n+m}\right)$, $\int \frac{u^{2}}{|y|^{2}}<\infty$ and

$$
\|u\|_{\mathcal{H}_{0}}^{2}=\int|\nabla u|^{2}-\left(\frac{m-2}{2}\right)^{2} \int \frac{u^{2}}{|y|^{2}} .
$$

Substitution of $u=w$ proves therefore that $\kappa_{m, n}<S_{N}$ whenever $m>2$. 
Case (ii). Let $z_{0}=\left(x_{0}, y_{0}\right) \in \mathbb{R}^{n+m} \backslash \mathbb{R}^{n}$ and let $\rho \in\left(0, \frac{|y|}{3}\right)$. Let $\psi \in C_{0}^{\infty}\left(B_{\rho}\left(z_{0}\right) ;[0,1]\right)$ be equal 1 on $B_{\rho}\left(z_{0}\right)$. These parameters will remain fixed. Let now $w_{\epsilon}=\epsilon^{-\frac{N-2}{2}} w\left((x-x+0) / \epsilon\left(y-y_{0}\right) / \epsilon\right)$. It suffices to prove that for $\epsilon$ sufficiently small,

$$
\frac{\int\left|\nabla\left(\psi w_{\epsilon}\right)\right|^{2}-\frac{1}{4} \int \frac{\left(\psi w_{\epsilon}\right)^{2}}{y^{2}}}{\left(\int\left(\psi w_{\epsilon}\right)^{2^{*}}\right)^{\frac{2}{2^{*}}}}<S_{N},
$$

since the left hand side is greater or equal to $\kappa_{1, n}$.

Note that $y$ is bounded from above and from below on $B_{\rho}\left(z_{0}\right)$, so it suffices to show that for every $\lambda>0$

$$
\frac{\int_{B_{\rho}\left(z_{0}\right)}\left|\nabla\left(\psi w_{\epsilon}\right)\right|^{2}-\lambda \int_{B_{\rho}\left(z_{0}\right)}\left(\psi w_{\epsilon}\right)^{2}}{\left(\int_{B_{\rho}\left(z_{0}\right)}\left(\psi w_{\epsilon}\right)^{2^{*}}\right)^{\frac{2}{2^{*}}}}<\frac{\int_{\mathbb{R}^{N}}\left|\nabla w_{\epsilon}\right|^{2}}{\left(\int_{\mathbb{R}^{N}} w_{\epsilon}^{2^{*}}\right)^{\frac{2}{p}}}=S_{N} .
$$

Verification of this is a literal repetition of the argument in (4), cases $N=4$ and $N>4$, and can be omitted.

Remark 2.3. Let $\Omega \subset \mathbb{R}^{n+m} \backslash \mathbb{R}^{n}$ be an open set. Set

$$
\kappa_{m, n}(\Omega):=\inf _{u \in C_{0}^{\infty}(\Omega): \int_{\mathbb{R}^{n+m}}|u|^{2^{*}}=1}\|u\|_{\mathcal{H}_{0}}^{2} .
$$

Then $\kappa_{m, n}(\Omega)<S_{N}$ whenever $m, n$ as in Lemma 2.2. The proof follows literally that of Lemma 2.3. part (ii), provided that the point $z_{0}$ is chosen in $\Omega$. Note that for $m>2, n \neq 0$ one has always $m+n>3$.

Definition 2.4. Let $H$ be a Hilbert space equipped with a group $G$ of bounded operators. We say that a sequence $u_{k} \in X$ converges to $u \in X G$-weakly, which we will denote as

$$
u_{k} \stackrel{G}{\rightarrow} u
$$

if for every sequence $g_{k} \in G$,

$$
g_{k}\left(u_{k}-u\right) \rightarrow 0 .
$$

Consider the following group acting on $\mathbb{R}^{n+m} \backslash \mathbb{R}^{n}$ :

$$
d:=\left\{\eta_{\alpha, j}:(x, y) \mapsto\left(2^{-j} x-\alpha, 2^{-j} y\right), j \in \mathbb{R}, \alpha \in \mathbb{R}^{n}\right\} .
$$

We associate with the group $d$ the following group of unitary operators on $\mathcal{H}_{0}$ :

$$
D_{0}:=\left\{g_{\alpha, j}: v \mapsto 2^{-j(N-2) / 2} v \circ \eta_{\alpha, j}, \eta_{\alpha, j} \in d\right\} .
$$

Operators in $D_{0}$ also preserve the $L^{2^{*}}$-norm.

By the isometry (5) $D=T^{-1} D_{0} T$ defines a group of unitary operators on $\mathcal{H}$ (which also preserve $\left.\int|y|^{2^{*}(1-m / 2)}|v|^{2^{*}}\right)$ :

$$
D:=\left\{g_{\alpha, j}: v \mapsto 2^{-j n / 2} v \circ \eta_{\alpha, j}, \eta_{\alpha, j} \in d\right\}
$$




\section{Penalty at infinity}

Lemma 3.1. Let $u_{k}$ be a bounded sequence in $\mathcal{H}_{0}$. If $\left|y_{k}\right| \rightarrow \infty$, then for all $k$ sufficiently large $u_{k}\left(\cdot+\left(0, y_{k}\right)\right)$ is bounded in $H_{l o c}^{1}\left(\mathbb{R}^{n+m}\right)$.

Proof. Let $\Omega \subset \mathbb{R}^{n+m}$ be an open bounded set. Then by the Hölder inequality

$$
\int_{\Omega}\left|y-y_{k}\right|^{-2}\left|u_{k}\left(\cdot+\left(0, y_{k}\right)\right)\right|^{2} \leq\left(\int_{\Omega}\left|u_{k}\left(\cdot+\left(0, y_{k}\right)\right)\right|^{2^{*}}\right)^{\frac{2}{2^{*}}}\left(\int_{\Omega}\left|y-y_{k}\right|^{-N}\right)^{\frac{2}{N}} .
$$

The first integral in the right hand side is bounded since $u_{k}$ is bounded in $\mathcal{H}_{0}$ and, therefore, by (21) in $L^{2^{*}}$. The expression under the second integral converges uniformly to zero. Therefore, the left hand side converges to zero, and consequently,

$$
\begin{array}{r}
C \geq \int_{\Omega}\left|y+y_{k}\right|^{2-m} \mid \nabla\left(\left.\left|y+y_{k}\right|^{\frac{m-2}{2}} u_{k}\left(\cdot+\left(0, y_{k}\right)\right)\right|^{2} \geq\right. \\
\frac{1}{2} \int_{\Omega}\left|\nabla u_{k}\left(\cdot+\left(0, y_{k}\right)\right)\right|^{2}-C \int_{\Omega}\left|y+y_{k}\right|^{-2} u_{k}\left(\cdot+\left(0, y_{k}\right)\right)^{2}= \\
\int_{\Omega} \frac{1}{2}\left|\nabla u_{k}\left(\cdot+\left(0, y_{k}\right)\right)\right|^{2}+o(1) .
\end{array}
$$

Therefore $\int_{\Omega} \frac{1}{2}\left|\nabla u_{k}\left(\cdot+\left(0, y_{k}\right)\right)\right|^{2}$ is bounded. It remains to note that $\| u_{k}\left(\cdot+\left(0, y_{k}\right)\left\|_{2^{*}}=\right\| u_{k} \|_{2^{*}}\right.$, which is bounded by the $\mathcal{H}_{0}$-norm.

We call the sequence $u_{k} \in \mathcal{H}_{0}$ (resp. $v_{k} \in \mathcal{H}$ ) a minimizing sequence, if $\left\|u_{k}\right\|_{2^{*}}=1$ and $\left\|u_{k}\right\|_{\mathcal{H}_{0}}^{2} \rightarrow \kappa_{m, n}$ (resp. $\left\|v_{k}\right\|_{2^{*},|y|^{2^{*}(1-m / 2)}}=1$ and $\left.\left\|v_{k}\right\|_{\mathcal{H}}^{2} \rightarrow \kappa_{m, n}\right)$.

Lemma 3.2. Assume that $\kappa_{m, n}<S_{N}$. If $u_{k} \in \mathcal{H}_{0}$ is a minimizing sequence and $\left|y_{k}\right| \rightarrow \infty$, then $u_{k}\left(\cdot+\left(0, y_{k}\right)\right) \rightarrow 0$ in $H_{l o c}^{1}\left(\mathbb{R}^{n+m} \backslash \mathbb{R}^{n}\right)$ and in $L^{2^{*}}\left(\mathbb{R}^{n+m}\right)$.

Proof. If the assertion of the lemma is false, then there is a $w \in L^{2^{*}} \backslash\{0\}$ and (taking into account Lemma 3.1) a renumbered subsequence such that $u_{k}\left(\cdot+\left(0, y_{k}\right)\right) \rightarrow w$ in $H_{l o c}^{1}$ and in $L^{2^{*}}$. Assume now that, on a renumbered subsequence, $\int\left|u_{k}\left(\cdot+\left(0, y_{k}\right)\right)-w\right|^{2^{*}} \rightarrow t \in[0,1]$.

Assume that $t \neq 1$. By the Brezis-Lieb lemma for $L^{2^{*}}\left(\mathbb{R}^{n+m}\right)([3])$,

$$
\int_{\mathbb{R}^{n+m}}|w|^{2^{*}}=1-t
$$

Let $w_{\epsilon} \in C_{0}^{\infty}\left(\mathbb{R}^{n+m} \backslash \mathbb{R}^{n}\right)$ be given by Lemma 2.1 and let $v_{k}^{\epsilon}:=u_{k}-w_{\epsilon}\left(\cdot-\left(0, y_{k}\right)\right)$. Observing that, since $w_{\epsilon}$ has compact support,

$$
\left.\left.\left|\int\right| y\right|^{-2} u_{k} w_{\epsilon}\left(\cdot-\left(0, y_{k}\right)\right)|=| \int\left|y+y_{k}\right|^{-2} u_{k} w_{\epsilon}\left|\leq C_{\epsilon}\right| y_{k}\right|^{-2}\left\|u_{k}\right\|_{\mathcal{H}_{0}} \rightarrow 0,
$$

and

$$
\int|y|^{-2} w_{\epsilon}\left(\cdot-\left(0, y_{k}\right)\right)^{2}=\int\left|y+y_{k}\right|^{-2} w_{\epsilon}^{2} \rightarrow 0
$$


we have the following estimate:

$$
\begin{array}{r}
\kappa_{m, n}=\left\|u_{k}\right\|_{\mathcal{H}_{0}}^{2}+o(1)= \\
\left\|v_{k}^{\epsilon}\right\|_{\mathcal{H}_{0}}^{2}+\int\left|\nabla w_{\epsilon}\right|^{2}+2 \int \nabla v_{k}^{\epsilon} \cdot \nabla w_{\epsilon}\left(\cdot-\left(0, y_{k}\right)\right)-2\left(\frac{m-2}{2}\right)^{2} \int|y|^{-2} v_{k}^{\epsilon} w_{\epsilon}\left(\cdot-\left(0, y_{k}\right)\right)+o(1)= \\
\left\|v_{k}^{\epsilon}\right\|_{\mathcal{H}_{0}}^{2}+\int\left|\nabla w_{\epsilon}\right|^{2}+2 \int \nabla u_{k} \cdot \nabla w_{\epsilon}\left(\cdot-\left(0, y_{k}\right)\right)+ \\
2\left(\frac{m-2}{2}\right)^{2} \int|y|^{-2} u_{k} w_{\epsilon}\left(\cdot-\left(0, y_{k}\right)\right)-2 \int\left|\nabla w_{\epsilon}\right|^{2}-2\left(\frac{m-2}{2}\right)^{2} \int\left|y+y_{k}\right|^{-2}\left|w_{\epsilon}\right|^{2}+o(1)= \\
\left\|v_{k}^{\epsilon}\right\|_{\mathcal{H}_{0}}^{2}+\int\left|\nabla w_{\epsilon}\right|^{2}+2 \int \nabla w \cdot \nabla w_{\epsilon}-\int\left|\nabla w_{\epsilon}\right|^{2}+o(1) \geq\left\|v_{k}^{\epsilon}\right\|_{\mathcal{H}_{0}}^{2}+(1-2 \epsilon) \int\left|\nabla w_{\epsilon}\right|^{2}+o(1) .
\end{array}
$$

Note that from this estimate follows that that $\int\left|\nabla w_{\epsilon}\right|^{2}$ is bounded from above uniformly in $\epsilon$, which implies that $w \in \mathcal{D}^{1,2}\left(\mathbb{R}^{N}\right)$. Then, we can use the second part of Lemma 2.1 and choose a $w_{\epsilon}$ so that, additionally, $\left\|w-w_{\epsilon}\right\|_{\mathcal{D}^{1,2}} \leq \epsilon$.

Consequently,

$$
\kappa_{m, n} \geq \lim \sup \left\|v_{k}^{\epsilon}\right\|_{\mathcal{H}_{0}}^{2}+(1-4 \epsilon) \int|\nabla w|^{2}
$$

By assumption, $S_{n}>\kappa_{m, n}$, so there exists an $\epsilon>0$ such that $(1-4 \epsilon) S_{n}>\kappa_{m, n}$. From (33) then follows:

$$
\kappa_{m, n}>\kappa_{m, n} t^{\frac{2}{2^{*}}}+\kappa_{m, n}(1-t)^{\frac{2}{2^{*}}},
$$

for all $t \in[0,1)$, which is false. Thus the assumption $t \neq 1$ is false and by (30), from $t=1$ follows $w=0$.

Remark 3.3. Let $\Omega \subset \mathbb{R}^{n+m} \backslash \mathbb{R}^{n}$ be an open set. The assertion of Lemma 3.2 holds also if $u_{k}$ is a minimizing sequence for $\kappa_{m, n}(\Omega)$. The only modification required for the proof is that inequality $\kappa_{m, n}(\Omega)<S_{N}$ (due to Remark 2.3) replaces $\kappa_{m, n}<S_{N}$.

Let $\chi \in C_{0}^{\infty}((0, \infty))$ be the following even function: $\chi(t)=0$ when $t \leq \frac{1}{2}$ or $t \geq 4, \chi(t)=2\left(t-\frac{1}{2}\right)$ when $t \in\left[\frac{1}{2}, 1\right], \chi(t)=t$ when $t \in[1,2], \chi(t)=\frac{1}{2}(4-t)$ when $t \in[2,4]$. Let

$$
\chi_{j}=2^{j} \chi\left(2^{-j}|\cdot|\right), j \in \mathbb{R} .
$$

Let

$$
B_{j}=\left(0,2^{j}\right)^{n} \times\left\{2^{j}<|y|<2^{j+1}\right\}, j \in \mathbb{R} .
$$

Lemma 3.4. Let $u_{k} \in \mathcal{H}_{0}$ be a bounded sequence. If $u_{k} \stackrel{D_{0}}{0}$ and,for every sequence $\left(x_{k}, y_{k}\right) \in \mathbb{R}^{n+m}$, $u_{k}\left(\cdot+\left(x_{k}, y_{k}\right)\right) \rightarrow 0$ in $H_{l o c}^{1}\left(\mathbb{R}^{n+m}\right)$, then for every sequence $j_{k} \in \mathbb{R}$

$$
\int_{B_{j_{k}}} \chi_{0}\left(u_{k}\right)^{2^{*}} \rightarrow 0 .
$$


Proof. It suffices to consider three cases: 1) $j_{k} \rightarrow-\infty$;2) $j_{k} \rightarrow+\infty$ and 3) $j_{k}$ is a bounded sequence.

Case 1. If $j_{k} \rightarrow-\infty, \int_{B_{j_{k}}} \chi_{0}\left(u_{k}\right)^{2^{*}} \leq C\left|B_{j_{k}}\right| \rightarrow 0$.

Case 2. Assume that $\left|j_{k}\right| \leq M \in \mathbb{R}$. Then $\int_{B_{j_{k}}} \chi_{0}\left(u_{k}\right)^{2^{*}} \rightarrow 0$ since $u_{k} \rightarrow 0$ in $\mathcal{D}_{\text {loc }}^{1,2}$, and in particular, $u_{k} \rightarrow 0$ locally in measure.

Case 3. Assume that $j_{k} \rightarrow+\infty$ and, without loss of generality, that $j_{k} \in \mathbb{N}$. Consider a tesselation of $B_{j_{k}}$ by the sets $B_{j_{k}}^{i l}=Q_{i} \times\left\{2^{j_{k}}+l<|y|<2^{j_{k}}+l+1\right\}$, where $Q_{i}$ are unit cubes in $\mathbb{R}^{n}$ and $l=0, \ldots, 2^{j_{k}+1}-1$. We will use the following version of the Sobolev inequality that holds for all $i, l$ with a uniform constant $C$ :

$$
\left(\int_{B_{j_{k}}^{i l}} w^{2^{*}}\right)^{\frac{2}{2^{*}}} \leq C\left(\int_{B_{j_{k}}^{i l}}|y|^{2-m}\left|\nabla\left(|y|^{\frac{m-2}{2}} w\right)\right|^{2}+\int_{B_{j_{k}}^{i l}}|y|^{-2}|w|^{2}\right) .
$$

Substituting $w=\chi_{0}\left(u_{k}\right)$ and taking into account that $\chi_{0}(t)^{2} \leq C t^{2^{*}}$ and that $|y|^{-2} \leq 2^{-j}$, we have, with a renamed constant,

$$
\int_{B_{j_{k}}^{i l}} \chi_{0}\left(u_{k}\right)^{2^{*}} \leq C\left(\int_{B_{j_{k}}^{i l}}|y|^{2-m}\left|\nabla\left(|y|^{\frac{m-2}{2}} u_{k}\right)\right|^{2}+\int_{B_{j_{k}}^{i l}}\left|u_{k}\right|^{2^{*}}\right)\left(\int_{B_{j_{k}}^{i l}} \chi_{0}\left(u_{k}\right)^{2^{*}}\right)^{1-\frac{2}{2^{*}}}
$$

Adding the inequalities above over all $i, l$, we get

$$
\int_{B_{j_{k}}} \chi_{0}\left(u_{k}\right)^{2^{*}} \leq C\left(\int_{B_{j_{k}}}|y|^{2-m}\left|\nabla\left(|y|^{\frac{m-2}{2}} u_{k}\right)\right|^{2}+\int_{B_{j_{k}}}\left|u_{k}\right|^{2^{*}}\right) \sup _{i, l}\left(\int_{B_{j_{k}}^{i l}} \chi_{0}\left(u_{k}\right)^{2^{*}}\right)^{1-\frac{2}{2^{*}}} .
$$

Note that the first factor in the right hand side is bounded by $\left\|u_{k}\right\|_{\mathcal{H}_{0}}$. Hence, in order to verify (37) it suffices to show that

$$
\int_{B_{0}} \chi_{0}\left(u_{k}\left(\cdot-\left(x_{k}, y_{k}\right)\right)\right)^{2^{*}} \rightarrow 0
$$

for all $\left(x_{k}, y_{k}\right) \in \mathbb{R}^{n+m}$ with $\left|y_{k}\right| \rightarrow \infty$. Indeed, since by assumption $u_{k}\left(\cdot-\left(x_{k}, y_{k}\right)\right) \rightarrow 0$ in $H^{1}\left(B_{0}\right)$,

$$
\int_{B_{0}}\left|u_{k}\left(\cdot-\left(x_{k}, y_{k}\right)\right)\right|^{2} \rightarrow 0 \text { for }\left|y_{k}\right| \rightarrow \infty .
$$

¿From here follows (41), and therefore, (37), once we take into account that $\chi(t)^{2^{*}} \leq C t^{2}$.

\section{Existence of the minimizer}

We start this section in interpreting the conclusion of Lemma 3.4 in terms of $\mathcal{H}, D$. The subsequent proofs will be carried out in the space $\mathcal{H}$. 
Lemma 4.1. Let $v_{k} \in \mathcal{H}$ be a bounded sequence such that for all sequences $\left(x_{k}, y_{k}\right) \in \mathbb{R}^{n+m}, t_{k}>0$,

$$
t_{k}^{\frac{N-2}{2}} T v_{k}\left(t_{k} \cdot+\left(x_{k}, y_{k}\right)\right) \rightarrow 0
$$

Then for every sequence $j_{k} \in \mathbb{Z}$

$$
\int_{B_{j_{k}}}|y|^{2^{*}(1-m / 2)} \chi_{0}\left(v_{k}\right)^{2^{*}} \rightarrow 0
$$

Proof. Let $j \in \mathbb{R}, v \in \mathcal{H}, u=T v$. Then, observing that $|y| \in\left(2^{j}, 2^{j+1}\right)$ on $B_{j}$,

$$
\begin{array}{r}
\int_{B_{j}}|y|^{2^{*}(1-m / 2)} \chi_{0}(v)^{2^{*}}= \\
\int_{B_{j}}|y|^{2^{*}(1-m / 2)} \chi_{0}\left(|y|^{\frac{m-2}{2}} u\right)^{2^{*}} \leq \\
C 2^{j 2^{*}(1-m / 2)}\left(\int_{B_{j}} \chi_{0}\left(2^{j \frac{m-2}{2}} u\right)^{2^{*}}+\int_{B_{j}} \chi_{0}\left(2^{(j+1) \frac{m-2}{2}} u\right)^{2^{*}}\right) .
\end{array}
$$

Let us estimate the first integral in the last expression. The estimate of the second integral is totally analogous and may be omitted. Let $t_{j}=2^{j \frac{m-2}{N-2}}$ and let $u^{j}=t_{j}^{\frac{N-2}{2}} u\left(t_{j^{*}}\right)$. Then

$$
2^{j 2^{*}(1-m / 2)} \int_{B_{j}} \chi_{0}\left(2^{j \frac{m-2}{2}} u\right)^{2^{*}} \leq \int_{B_{\frac{n j}{N-2}}} \chi_{0}\left(u^{j}\right) .
$$

Let now $j_{k}$ be an arbitrary sequence and substitute $j=j_{k}, u=u_{k}:=T v_{k}$ :

$$
2^{j_{k} 2^{*}(1-m / 2)} \int_{B_{j_{k}}} \chi_{0}\left(2^{j_{k} \frac{m-2}{2}} u_{k}\right)^{2^{*}} \leq \int_{B_{j_{k} \frac{n}{N-2}}} \chi_{0}\left(u_{k}^{j_{k}}\right),
$$

where $u_{k}^{j_{k}}:=t_{j_{k}}^{\frac{N-2}{2}} u\left(t_{j_{k}} \cdot\right)$ still satisfies the assumptions of the lemma and thus the assumptions of Lemma 3.4. From the latter follows that the right hand side in (47) converges to zero, and tracing back (45) with $u=u_{k}=T v_{k}, j=j_{k}$, we arrive at (44).

Lemma 4.2. If $v_{k} \in \mathcal{H}$ is as in Lemma 4.1, then $v_{k} \rightarrow 0$ in $L^{2^{*}}\left(\mathbb{R}^{n+m},|y|^{2^{*}(1-m / 2)}\right)$.

Proof. Let us use the following version of Sobolev inequality with a fixed $q \in\left(2,2^{*}\right)$ :

$$
C\left(\int_{B_{0}}|y|^{2^{*}(1-m / 2)}|v|^{2^{*}}\right)^{\frac{q}{2^{*}}} \leq \int_{B_{0}}|y|^{2-m}|\nabla v|^{\frac{q}{2}}+\int_{B_{0}}|y|^{n q / 2-N}|w|^{q} .
$$

The exponent $n q / 2-N$ is chosen so that the integral of the respective expression over the whole $\mathbb{R}^{n+m} \backslash \mathbb{R}^{n}$ is dilation invariant, so that the inequality holds (with the same constant) with $B_{0}$ replaced with $\eta_{\alpha, j} B_{0}$, for all $j \in \mathbb{Z}, \alpha \in \mathbb{R}^{n}$. Substituting $v=\chi_{i}\left(v_{k}\right), i \in \mathbb{Z}$, we get 


$$
\begin{array}{r}
\int_{\eta_{\alpha, j} B_{0}}|y|^{2^{*}(1-m / 2)} \chi_{i}\left(v_{k}\right)^{2^{*}} \leq \\
C\left(\int_{\eta_{\alpha, j} B_{0} \cap \operatorname{supp} \chi_{i}\left(v_{k}\right)}|y|^{2-m}\left|\nabla v_{k}\right|^{\frac{q}{2}}+\int_{\eta_{\alpha, j} B_{0}}|y|^{n q / 2-N} \chi_{i}\left(v_{k}\right)^{q}\right) \times\left(\int_{\eta_{\alpha, j} B_{0}} \chi_{i}\left(v_{k}\right)^{2^{*}}\right)^{1-\frac{q}{2^{*}}} .
\end{array}
$$

Adding terms up over all $i, j \in \mathbb{Z}$ and $\alpha \in \mathbb{Z}^{n}$, we have

$$
\begin{gathered}
\int_{\mathbb{R}^{n+m}}|y|^{2^{*}(1-m / 2)}\left|v_{k}\right|^{2^{*}} \leq C\left(\int_{\mathbb{R}^{n+m}}|y|^{2-m}\left|\nabla v_{k}\right|^{\frac{q}{2}}+\int_{\mathbb{R}^{n+m}}|y|^{n q / 2-N}\left|v_{k}\right|^{q}\right) \times \\
\sup _{i, j, \alpha}\left(\int_{\eta_{\alpha, j} B_{0}}|y|^{2^{*}(1-m / 2)} \chi_{i}\left(v_{k}\right)^{2^{*}}\right)^{1-\frac{q}{2^{*}}}
\end{gathered}
$$

Note that the first factor in the last expression is bounded, since $v_{k}$ is a bounded sequence in $\mathcal{H}$. In particular,

$$
\int_{\mathbb{R}^{n+m}}|y|^{n q / 2-N}\left|v_{k}\right|^{q} \leq C\left\|v_{k}\right\|_{\mathcal{H}}^{q}
$$

due to the correspondent inequality $([13]$, p.98, Corollary 3. Thus the lemma is proved once we verify that for an arbitrary sequence $i_{k}, j_{k} \in \mathbb{Z}$ and $\alpha_{k} \in \mathbb{Z}^{n}$,

$$
\int_{\eta_{j_{k}, \alpha_{k}} B_{0}}|y|^{2^{*}(1-m / 2)} \chi_{i_{k}}\left(v_{k}\right)^{2^{*}} \rightarrow 0 .
$$

This, however, is an immediate corollary of (44), once we substitute $v_{k}=t_{k}^{\frac{n}{2}} \tilde{v}_{k}\left(t_{k} \cdot\right)$ with a suitable sequence $t_{k}$.

Corollary 4.3. Let $v_{k}$ be a minimizing sequence for (7), namely, $\left\|v_{k}\right\|_{\mathcal{H}}^{2} \rightarrow \kappa_{m, n},\left\|v_{k}\right\|_{2^{*} ;|y|^{2^{*}(1-m / 2)}}=$ 1. Then there is a sequence $g_{k} \in D$, such that, on a renamed subsequence, w-lim $g_{k} v_{k} \neq 0$.

Proof. Assume the opposite, namely that $v_{k} \stackrel{D}{0} 0$. Note that $v_{k}$ is a minimizing sequence, and so is $g_{k} v_{k}$ with any $g_{k} \in D$. Then by Lemma 3.2 the sequence $u_{k}=T v_{k}$ satisfies the assumptions of Lemma 4.2 and thus $v_{k} \rightarrow 0$ in $L^{2^{*}}\left(\mathbb{R}^{n+m},|y|^{2^{*}(1-m / 2)}\right)$, a contradiction.

We now can prove Theorem 1.1, from which Theorem 1.2 follows immediately due to the isometry (15).

Proof of Theorem 1.1. Let $v_{k}$ be a minimizing sequence. Due to Corollary 4.3, we may assume without loss of generality that $v_{k} \rightarrow w \neq 0$. Then $t:=\int_{\mathbb{R}^{n+m}}|w|^{2^{*}}|y|^{2^{*}(1-m / 2)} \in(0,1]$. From Brezis-Lieb lemma follows then that $\int_{\mathbb{R}^{n+m}}\left|v_{k}-w\right|^{2^{*}}|y|^{2^{*}(1-m / 2)}=1-t$. Therefore,

$$
\kappa_{m, n}=\lim \left\|v_{k}\right\|_{\mathcal{H}}^{2}=\|w\|_{\mathcal{H}}^{2}+\lim \left\|v_{k}-w\right\|_{\mathcal{H}}^{2} \geq \kappa_{m, n} t^{\frac{2}{2^{*}}}+\kappa_{m, n}(1-t)^{\frac{2}{2^{*}}}
$$

This inequality holds only as equality at the endpoints $t=0,1$ and thus, with necessity, $t=1$. In other words, $v_{k} \rightarrow w$ in $L^{2^{*}}\left(\mathbb{R}^{n+m},|y|^{2^{*}(1-m / 2)}\right)$ and therefore $w$ is a minimizer. 


\section{$5 \quad$ Existence and non-existence for $\Omega \subset \mathbb{R}^{n+m} \backslash \mathbb{R}^{n}$}

In this section we give the proofs of Theorem 1.3 and Theorem 1.4.

Proof of Theorem 1.3. Let $u_{\epsilon} \in C_{0}^{\infty}\left(\mathbb{R}^{n+m} \backslash \mathbb{R}^{n}\right), \epsilon>0$, satisfy

$$
\|u\|_{2^{*},|y|^{2^{*}(1-m / 2)}}=1 \text { and }\left\|u_{\epsilon}\right\|_{\mathcal{H}}^{2} \leq \kappa_{m, n}+\epsilon
$$

and set $v_{\epsilon, t}:=t^{\frac{N-2}{2}} u_{\epsilon}(t \cdot), t>0$.

Case (a): Without loss of generality assume that $x_{0}=0$. Then for $t>0$ sufficiently large $v_{\epsilon, t} \in C_{0}^{\infty}\left(B_{r}(0)\right)$ and still satisfies (53). Consequently, $\kappa_{m, n}\left(B_{r}(0)\right) \leq \kappa_{m, n}+\epsilon$, and since $\epsilon$ is arbitrary, $\left.\kappa_{m, n}\left(B_{r}(0)\right)\right) \leq \kappa_{m, n}$. The converse inequality $\kappa_{m, n}\left(B_{r}(0)\right) \geq \kappa_{m, n}$ is immediate.

Case (b): The proof is completely analogous to the case (a) once we note that for $t>0$ sufficiently small $v_{\epsilon, t} \in C_{0}^{\infty}(\{|y|>R\})$.

We proceed now with the proof of Theorem 1.4. The following statement is a particular case of the global compactness theorem from [17] to the case $\mathcal{D}^{1,2}\left(\mathbb{R}^{N}\right)$ with the group $D_{1}$ of unitary operators generated by actions of dilations

$$
\left(h_{t} u\right)(x)=t^{\frac{N-2}{2}} u(t x), t>0,
$$

and translations

$$
u \mapsto u(\cdot-z), z \in \mathbb{R}^{N} .
$$

Theorem 5.1. Let $u_{k} \in \mathcal{D}^{1,2}\left(\mathbb{R}^{N}\right)$ be a bounded sequence. Then there exists $w^{(\ell)} \in \mathcal{D}^{1,2}\left(\mathbb{R}^{N}\right)$, $g_{k}^{(\ell)} \in D_{1}, k, \ell \in \mathbb{N}$, such that for a renumbered subsequence one has:

$$
\begin{aligned}
g_{k}^{(1)} & =i d, g_{k}^{(i)^{-1}} g_{k}^{(j)} \rightarrow 0 \text { for } i \neq j, \\
w^{(\ell)} & =\mathrm{w}-\lim g_{k}^{(\ell)^{-1}} u_{k} \\
\sum_{\ell \in \mathbb{N}}\left\|w^{(\ell)}\right\|^{2} & \leq \limsup \left\|u_{k}\right\|^{2} \\
u_{k}-\sum_{\ell \in \mathbb{N}} g_{k}^{(\ell)} w^{(\ell)} & \rightarrow 0 \text { in } L^{2^{*}}\left(\mathbb{R}^{N}\right) .
\end{aligned}
$$

The series in (59) is absolutely convergent in $\mathcal{D}^{1,2}$, uniformly in $k$.

Lemma 5.2. Let $u_{k}, w^{(i)}$ be as in Theorem 5.1. Then

$$
\int_{\mathbb{R}^{N}}\left|u_{k}\right|^{2^{*}} \rightarrow \sum_{i} \int_{\mathbb{R}^{N}}\left|w^{(i)}\right|^{2^{*}}
$$

Proof. By (59) and continuity of $u \mapsto \int_{\mathbb{R}^{N}}|u|^{2^{*}}$ in $\mathcal{D}^{1,2}\left(\mathbb{R}^{N}\right)$ it suffices to prove the lemma for $u_{k}^{M}:=\sum_{i=1}^{M} g_{k}^{(i)} w^{(i)}, M \in \mathbb{N}$. Iterating Brezis-Lieb lemma for $M-1$ steps, we obtain immediately

$$
\int_{\mathbb{R}^{N}}\left|u_{k}^{M}\right|^{2^{*}}=\sum_{i=1}^{M} \int_{\mathbb{R}^{N}}\left|w^{(i)}\right|^{2^{*}}
$$


Lemma 5.3. Let $\Omega \subsetneq \mathbb{R}^{N}$ be an open set with $\partial \Omega \in C^{1}$ and let $u_{k} \in H_{0}^{1}(\Omega)$. If there exist $t_{k}>0$, $z_{k} \in \mathbb{R}^{N}, w \in \mathcal{D}^{1,2}\left(\mathbb{R}^{N}\right)$ such that $t_{k}^{-\frac{N-2}{2}} u_{k}\left(t_{k}^{-1} \cdot+z_{k}\right) \rightarrow w$ in $\mathcal{D}^{1,2}\left(\mathbb{R}^{N}\right)$, then, modulo a set of measure zero,

$$
V(w):=\left\{z \in \mathbb{R}^{N}: w(z) \neq 0\right\} \subset \liminf t_{k}\left(\Omega-z_{k}\right) .
$$

Moreover, if there exist $t_{0}>0$ and $z_{0} \in \mathbb{R}^{N}$ such that $\liminf t_{k}\left(\Omega-z_{k}\right) \subset t_{0}\left(\Omega+z_{0}\right)$, then $w\left(t_{0}^{-1}\left(\cdot-z_{0}\right)\right) \in H_{0, l o c}^{1}(\Omega)$.

Proof. Convergence $t_{k}^{-\frac{N-2}{2}} u_{k}\left(t_{k}^{-1} \cdot+z_{k}\right) \rightarrow w$ in $\mathcal{D}^{1,2}\left(\mathbb{R}^{N}\right)$ implies convergence a.e. Therefore, in the complement of a set of measure zero, $w(z)=0$ for any $z$ that is not in $\cap_{k \geq k_{0}}\left(t_{k}\left(\Omega-z_{k}\right)\right)$ for some $k_{0} \in \mathbb{N}$. Consequently, $w(z)=0$ unless, modulo a set of zero measure, $z \in \lim \inf \left(t_{k}\left(\Omega-z_{k}\right)\right)$. Then, by assumption, $V\left(w\left(t_{0}^{-1}\left(\cdot-z_{0}\right)\right) \subset \Omega\right.$. Since $\partial \Omega \in C^{1}$ and $w \in \mathcal{D}^{1,2}\left(\mathbb{R}^{N}\right)$, the conclusion $w\left(t_{0}^{-1}\left(\cdot-z_{0}\right)\right) \in H_{0, l o c}^{1}(\Omega)$ follows.

Proof of Theorem 1.4.

1. Observe that the norms $\mathcal{H}_{0}(\Omega), \mathcal{D}^{1,2}(\Omega)$ and $H_{0}^{1}(\Omega)$ are equivalent. The last two are equivalent by the Friedrichs inequality, which holds since $\partial \Omega \in C^{1}$ and $\sup _{x \in \Omega} d\left(x, \mathbb{R}^{N} \backslash \Omega\right)<\infty$. Since $\|u\|_{\mathcal{H}_{0}(\Omega)} \leq\|u\|_{\mathcal{D}^{1,2}(\Omega)}$, it suffices to show that

$$
C(\Omega):=\inf _{u \in C_{0}^{\infty}(\Omega): \int_{\Omega} \frac{u^{2}}{\left|y^{2}\right|}=1} \int_{\Omega}|\nabla u|^{2}>\left(\frac{m-2}{2}\right)^{2} .
$$

Let $R>0$ be such that $\Omega \subset A_{1 / R, R}$. Then it suffices to show that $C\left(A_{1 / R, R}\right)>\left(\frac{m-2}{2}\right)^{2}$. This easily follows from

$$
\inf _{u \in C_{0}^{\infty}\left(\omega_{R}\right): \int_{\omega_{R}} \frac{u^{2}}{\left|y^{2}\right|} d y=1} \int_{\omega_{R}}\left|\nabla_{y} u\right|^{2} d y>\inf _{u \in C_{0}^{\infty}\left(\mathbb{R}^{m}\right): \int_{\mathbb{R}^{m}} \frac{u^{2}}{\left|y^{2}\right|} d y=1} \int_{\mathbb{R}^{m}}\left|\nabla_{y} u\right|^{2} d y=\left(\frac{m-2}{2}\right)^{2},
$$

where $\omega_{R}=A_{1 / R, R} \cap \mathbb{R}^{m}$, while the latter inequality holds true since the minimum in the left hand side is attained (the Dirichlet problem on a bounded domain) and the minimizer cannot be a minimizer on $\mathbb{R}^{m}$ by the maximum principle.

2. Let $u_{k}$ be a minimizing sequence for (17). By the preceding step $u_{k}$ is bounded in $\mathcal{D}^{1,2}\left(\mathbb{R}^{N}\right)$. Assume that, on a renumbered subsequence,

$$
t_{k}^{-\frac{N-2}{2}} u\left(t_{k} \cdot+z_{k}\right) \rightarrow w \neq 0, z_{k}=\left(x_{k}, y_{k}\right), t_{k}>0
$$

Note that if $t_{k} \rightarrow 0$, the scaling argument gives $t_{k}-\frac{N-2}{2} u_{k}\left(t_{k} \cdot+z_{k}\right) \rightarrow 0$ in $L^{2}\left(\mathbb{R}^{N}\right)$, so $w=0$. If, on the other hand, there is a subsequence where both $t_{k}$ and $1 / t_{k}$ are bounded, but $y_{k}$ is unbounded, from Lemma 5.3 follows that the set $\{w \neq 0\}$ has measure zero, which also yields $w=0$. We conclude that $w \neq 0$ only if either (a) $t_{k} \rightarrow \infty$ or (b) $t_{k}, 1 / t_{k}$ and $y_{k}$ are bounded.

3. Let us show now that case (a) does not occur. Assume that there is a sequence $\left(z_{k}, t_{k}\right) \in$ $\mathbb{R}^{N} \times(0, \infty), t_{k} \rightarrow \infty$, such that, on a renamed subsequence, $t_{k}^{-\frac{N-2}{2}} u_{k}\left(t_{k}^{-1} \cdot+z_{k}\right) \rightarrow w$ with some $w \in \mathcal{D}^{1,2}\left(\mathbb{R}^{N}\right) \backslash\{0\}$. Since $C_{0}^{\infty}\left(\mathbb{R}^{N}\right)$ is dense in $\mathcal{D}^{1,2}\left(\mathbb{R}^{N}\right)$, for every $\epsilon \in\left(0, \frac{1}{2}\right)$ there exists a $w_{\epsilon} \in C_{0}^{\infty}\left(\mathbb{R}^{N}\right)$ such that

$$
\left\|w_{\epsilon}-w\right\|_{\mathcal{D}^{1,2}} \leq \epsilon\|w\|_{\mathcal{D}^{1,2}}
$$


Moreover, we can choose $w_{\epsilon}$ so that for all $k$ sufficiently large $w_{\epsilon}\left(t_{k}\left(\cdot-z_{k}\right)\right)$ is supported in $\Omega+B_{2 \epsilon}(0)$. Indeed, $w_{\epsilon}\left(t_{k}\left(\cdot-z_{k}\right)\right)$ is supported in an arbitrarily small (for $k$ large) neighborhood of $z_{k}$ and, since for every $z \in \mathbb{R}^{N}, u_{k}\left(t_{k}^{-1} z+z_{k}\right)=0$ whenever $z_{k} \notin \Omega+B_{\epsilon}(0)$ and $k$ is sufficiently large, we have necessarily $z_{k} \in \Omega+B_{\epsilon}(0)$.

Let

$$
v_{\epsilon, k}=u_{k}-t_{k}^{\frac{N-2}{2}} w_{\epsilon}\left(t_{k}\left(\cdot-z_{k}\right)\right) .
$$

Then, using the scaling invariance of the involved integrals and reserving the norm notation for the $\mathcal{H}_{0}$-norm, one has

$$
\begin{array}{r}
\left\|u_{k}\right\|^{2}=\left\|v_{\epsilon, k}+t_{k}^{\frac{N-2}{2}} w_{\epsilon}\left(t_{k}\left(\cdot-z_{k}\right)\right)\right\|^{2}= \\
\left.\left\|v_{\epsilon, k}\right\|^{2}+\left\|t_{k}^{\frac{N-2}{2}} w_{\epsilon}\left(t_{k}\left(\cdot-z_{k}\right)\right)\right\|^{2}+2 \int \nabla v_{\epsilon, k} \cdot \nabla t_{k}^{\frac{N-2}{2}} w_{\epsilon}\left(t_{k}\left(\cdot-z_{k}\right)\right)\right) \\
\left.2\left(\frac{m-2}{2}\right)^{2} \int v_{\epsilon, k} t_{k}^{\frac{N-2}{2}} w_{\epsilon}\left(t_{k}\left(\cdot-z_{k}\right)\right)\right)|y|^{-2}= \\
\left\|v_{\epsilon, k}\right\|^{2}+\int\left|\nabla w_{\epsilon}\right|^{2}+o(1)+2 \int \nabla u_{k} \cdot \nabla t_{k}^{\frac{N-2}{2}} w_{\epsilon}\left(t_{k}\left(\cdot-z_{k}\right)\right)-2 \int\left|\nabla w_{\epsilon}\right|^{2}- \\
2\left(\frac{m-2}{2}\right)^{2} \int u_{k} t_{k}^{\frac{N-2}{2}} w_{\epsilon}\left(t_{k}\left(\cdot-z_{k}\right)\right)|y|^{-2}+2\left(\frac{m-2}{2}\right)^{2} \int t_{k}^{N-2}\left|w_{\epsilon}\left(t_{k}\left(\cdot-z_{k}\right)\right)\right|^{2}|y|^{-2}
\end{array}
$$

At the last step we have used the following estimates:

$$
\int t_{k}^{N-2}\left|w_{\epsilon}\left(t_{k}\left(\cdot-z_{k}\right)\right)\right|^{2}|y|^{-2}=t_{k}^{-2} \int\left|w_{\epsilon}\right|^{2}|y|^{-2}=o(1)
$$

and, by Cauchy inequality,

$$
\left.\left|\int u_{k} t_{k}^{\frac{N-2}{2}} w_{\epsilon}\left(t_{k}\left(\cdot-z_{k}\right)\right)\right| y\right|^{-2} \mid \leq\left(\int u_{k}^{2}|y|^{-2}\right)^{\frac{1}{2}}\left(\int t_{k}^{N-2}\left|w_{\epsilon}\left(t_{k}\left(\cdot-z_{k}\right)\right)\right|^{2}|y|^{-2}\right)^{\frac{1}{2}}=o(1) .
$$

Consequently, using (66), we obtain

$$
\kappa_{m, n}(\Omega)=\lim \left\|u_{k}\right\|^{2} \geq \liminf \left\|v_{\epsilon, k}\right\|^{2}+(1-8 \epsilon) \int|\nabla w|^{2} .
$$

From the definitions of $\kappa_{m, n}(\Omega)$ and $S_{N}$ then follows

$$
\kappa_{m, n}(\Omega) \geq \kappa_{m, n}(\Omega) \lim \inf \left\|v_{\epsilon, k}\right\|_{2^{*}}^{2}+(1-8 \epsilon) S_{N}\|w\|_{2^{*}}^{2} .
$$

Let now

$$
v_{k}=u_{k}-t_{k}^{\frac{N-2}{2}} w\left(t_{k}\left(\cdot-z_{k}\right)\right)
$$


Then (66) and (71) imply

$$
\kappa_{m, n}(\Omega) \geq \kappa_{m, n}(\Omega) \liminf \left\|v_{k}\right\|_{2^{*}}^{2}+(1-10 \epsilon) S_{N}\|w\|_{2^{*}}^{2},
$$

and, since $\epsilon$ is arbitrary,

$$
1 \geq \liminf \left\|v_{k}\right\|_{2^{*}}^{2}+\frac{S_{N}}{\kappa_{m, n}(\Omega)}\|w\|_{2^{*}}^{2}
$$

At the same time, from the Brezis-Lieb lemma 3] (passing to a renamed subsequence if necessary) follows

$$
\liminf \left\|v_{k}\right\|_{2^{*}}^{2^{*}}+\|w\|_{2^{*}}^{2^{*}}=1 .
$$

Since $S_{N}>\kappa_{m, n}(\Omega)$ by Remark 2.3 and $2^{*}>2$, relations (75) and (74) hold simultaneously only if $w=0$, a contradiction.

4. We conclude that case (b) is the only possibility for a non-zero weak limit (65). Consequently, for a renumbered subsequence, one can write (59) as

$$
u_{k}-\sum w^{(i)}\left(\cdot-x_{k}^{(i)}\right) \rightarrow 0 \text { in } L^{p}\left(\mathbb{R}^{N}\right), p \in\left(2,2^{*}\right]
$$

$\left|x_{k}^{(i)}-x_{k}^{(j)}\right| \rightarrow \infty$ for $i \neq j$, and, moreover (by using (15), Lemma 5.3 and the fact that $\mathcal{D}^{1,2}(\Omega)$ )-norm and the $\left.\mathcal{H}_{0}(\Omega)\right)$ are equivalent, $w^{(j)} \in H_{0}^{1}(\Omega)$. ¿From Lemma 5.2 follows that

$$
\int\left|u_{k}\right|^{2^{*}} \rightarrow \sum_{j} \int\left|w^{(j)}\right|^{2^{*}}=1
$$

while (58) and definition (12) of $\kappa_{m, n}(\Omega)$ imply

$$
\kappa_{m, n}(\Omega) \geq \sum_{j} \kappa_{m, n}(\Omega)\left(\int\left|w^{(j)}\right|^{2^{*}}\right)^{2 / 2^{*}} .
$$

Relations (77) and (78) are contradictive unless all but one $w^{(j)}$ equal zero and for some $j_{0}$, $\int\left|w^{\left(j_{0}\right)}\right|^{2^{*}}=1$. Then, necessarily, $w^{\left(j_{0}\right)}$ is a minimizer.

\section{Existence in $R^{n+m}$ - concluding remarks}

One can use the rearrangement argument to reduce the proof of Theorem 1.1] in the case $m>2$ to an existence result of Badiale and Tarantello [1]. It should be noted that this reduction does not extend to the case $m=1$, while the proof in the present paper is uniform with regard to $m$ and uses techniques that allow to approach analogous problems with lack of radial symmetry.

Proof. By the rearrangement argument, the minimum in (8) is attained in the subspace $\mathcal{H}_{r}$ of $\mathcal{H}$ of functions that are radially symmetric in the variable $y \in \mathbb{R}^{n}$, so we may restate the problem, regarding $|y|$ as a radial variable in $\mathbb{R}^{2}$, in the form

$$
\kappa_{m, n}=\inf _{v \in \mathcal{D}_{r}^{1,2}\left(\mathbb{R}^{n+2}\right): \frac{\omega_{m-1}}{2 \pi} \int_{R^{n} \times \mathbb{R}^{2}}|v(x, y)|^{\frac{2 N}{N-2}}|y|^{-\frac{2(m-2)}{N-2}} d x d y=1} \frac{\omega_{m-1}}{2 \pi} \int_{\mathbb{R}^{n} \times \mathbb{R}^{2}}|\nabla u(x, y)|^{2} d x d y
$$


We note that the exponent $\frac{2 N}{N-2}$ is subcritical in the dimension $n+2<N$ and we may apply Theorem 2.5 of [1], relative to $\mathbb{R}^{n+2}$. Parameters for the application, in the original notations of [1], are $q=2, s=\frac{2(m-2)}{N-2}$ and $q_{*}=\frac{2 N}{N-2}$.

Several related problems remain unresolved in this paper.

1. Evaluate the best constant $\kappa_{m, n}$ and find minimizers of (2) when the minimum exists. Is the inequality $\kappa_{1,2}<S_{3}$ true? If it is false, is there still a minimizer for $\kappa_{1,2}$ ?

2. We saw that when $\kappa_{m, n}(\Omega)=\kappa_{m, n}, \Omega \neq \mathbb{R}^{n+m} \backslash \mathbb{R}^{n}$, there is no minimizer for $\kappa_{m, n}(\Omega)$. Is the converse true? That is, does $\kappa_{m, n}(\Omega)>\kappa_{m, n}$ imply existence of the minimizer?

3. More general results of the form (2) were recently established ([14, 15]). For example if $\Omega$ is a bounded smooth and convex domain, $d(x)=\operatorname{dist}(x, \partial \Omega)$ then there exist a positive constant $C$ dependent on $\Omega$ such that

$$
\int_{\Omega}|\nabla u|^{2} d x-\frac{1}{4} \int_{\Omega} \frac{u^{2}}{d^{2}} d x \geq C\left(\int_{\Omega}|u|^{\frac{2 N}{N-2}} d x\right)^{\frac{N-2}{N}}, \quad \forall u \in C_{0}^{\infty}(\Omega) .
$$

We believe that the following is an interesting question. Is the best constant $C=C(\Omega)$ connected with the constant $\kappa_{1, n}$ ? In particular, is it true for convex $\Omega$ that $C(\Omega)=\kappa_{1, n}$ ?

4. By analogy with Theorem [1.1 it is natural to ask whether the following minimization problem

$$
\kappa_{m, n}^{p}=\inf _{\int_{\mathbb{R}^{n+m}}|y|^{\frac{N(p-m)}{N-p}}|v|^{\frac{N p}{N-p}} d x d y=1} \int_{\mathbb{R}^{n+m} \backslash \mathbb{R}^{n}}|y|^{-(m-p)}|\nabla v|^{p} d x d y
$$

has a minimizer when $m \neq p>1$, in particular when $p^{2}<N$. A partial answer can be given by an argument similar to the previous section, by Theorem 2.5 of [1].

\section{References}

[1] Badiale M., Tarantello G., Sobolev-Hardy Inequality with Applications to a Nonlinear Elliptic Equation arising in Astrophysics, Arch. Rational Mech. Anal. 163 (2002) 259-293

[2] Brezis H., Some variational problems with lack of compactness, in: Nonlinear functional analysis and its applications, Part 1 (Berkeley, Calif., 1983), 165-201, Proc. Sympos. Pure Math., 45, Part 1, Amer. Math. Soc., Providence, RI, 1986.

[3] Brezis H., Lieb E., A relation between pointwise convergence of functions and convergence of functionals, Proc. Amer. Math. Soc. 88 (1983) 486-490.

[4] Brezis, H., Nirenberg, L., Positive solutions of nonlinear elliptic equations involving critical Sobolev exponents, Comm. Pure Appl. Math. 36 (1983), 437-477.

[5] Brezis H., Vázquez J. L., Blowup solutions of some nonlinear elliptic problems, Revista Mat. Univ. Complutense Madrid 10 (1997), 443-469.

[6] Caffarelli, L., Kohn, R., Nirenberg, L., First order interpolation inequalities with weights. Compositio Math. 53 (1984), 259-275. 
[7] Lieb E.H., Sharp constants in the Hardy-Littlewood-Sobolev and related inequalities, Ann.Math.118 (1983), 349-374.

[8] Lions P.-L., The concentration-compactness principle in the calculus of variations. The locally compact case, part 1. Ann.Inst.H.Poincare, Analyse non linéaire 1, 109-1453 (1984)

[9] Lions P.-L., The concentration-compactness principle in the calculus of variations. The locally compact case, part 2. Ann.Inst.H.Poincare, Analyse non linéaire 1, 223-283 (1984)

[10] Lions P.-L., The concentration-compactness principle in the calculus of variations. The Limit Case, Revista Matematica Iberoamericana, Part 1, 1.1 145-201 (1985)

[11] Lions P.-L., The concentration-compactness principle in the calculus of variations. The Limit Case, Revista Matematica Iberoamericana, Part 2, 1.2 45-121 (1985)

[12] Markus M., Mizel V.J., Pinchover Y., On the best constant for Hardy's inequality in $\mathbb{R}^{n}$, Trans.Amer.Math.Soc. 350 (1998), 3237-3255.

[13] Maz'ya V.G, Sobolev Spaces, Springer Verlag 1985

[14] Filippas S., Maz'ya V. G. and Tertikas A., Sharp Hardy-Sobolev inequalities. (English. English, French summary) C. R. Math. Acad. Sci. Paris 339 (2004), 483-486.

[15] Filippas S., Maz'ya V. G. and Tertikas A., Critical Hardy-Sobolev inequalities, in preparation.

[16] Filippas S. and Tertikas A. Optimizing Improved Hardy inequalities. J. Funct. Anal. 192 (2002) 186-233.

[17] Schindler I., Tintarev K., An abstract version of the concentration compactness principle, Rev. Mat. Complut. 15 (2002), 417-436.

[18] Schindler I., Tintarev K., Abstract concentration compactness and semilinear elliptic equations on unbounded domains, in: M.R. Grossinho, M. Ramos, C. Rebelo, L. Sanchez, (Eds.), Progress in Nonlinear Differential Equations and Their Applications 43, Birkhuser Boston (2001), 369378.

[19] Tintarev K., Singular semilinear elliptic equations in the half-space, Rend. Istit. Mat. Univ. Trieste 33 (2001), 327-337. 